\title{
Prognostic factors of cervical node status in head and neck squamous cell carcinoma
}

\author{
Chairat Burusapat $^{1 *}$, Weerawut Jarungroongruangchai ${ }^{1}$ and Mongkon Charoenpitakchai ${ }^{2}$
}

\begin{abstract}
Background: Cervical nodal status is one of prognostic factors in head and neck squamous cell carcinoma (HNSCC). The objective of this study was to identify prognostic factors of cervical node status including site and size of primary tumors, presence of lymphovascular invasion, and size of cervical node for appropriate further treatment in HNSCC.

Methods: A 5-year retrospective review of patients with HNSCC in Phramongkutklao Hospital from 2009 to 2013 was conducted. Histopathologic data on primary tumors and cervical nodes were reviewed. Cervical nodes were divided into five groups: $1-3,4-6,7-9,10-30$, and $>30 \mathrm{~mm}$. Numbers of positive and negative nodes were compared in different sizes and sites and the presence of extracapsular extension.

Results: In all, 165 patients and 1,472 nodes were reviewed. The mean age was 52.6 years and $77.58 \%$ were male. The most frequent primary site was oral tongue (50.91\%). In sum, 52.72\% showed lymphovascular invasion.

Thirty-five patients (81.40\%) in therapeutic neck dissections and 18 patients (69.23\%) in prophylactic neck dissections showed nodal metastasis. The mean size of metastatic nodes was $3.89 \mathrm{~mm}$ (range, 2-45 mm) and $3.53 \mathrm{~mm}$ (range, 2-23 mm), respectively. Significant associations were found between the size of cervical nodes and the site of primary tumor of the oral tongue, lip, base of the tongue, and floor of the mouth $(p<0.05)$. Metastatic lymph nodes showed extracapsular extension $69.55 \%$. No significance was found between extracapsular extension and clinical staging, size of primary tumor, pathologic differentiation, and size of cervical nodes. Sizes of cervical lymph node of squamous cell carcinoma (SCC) of the oral tongue and lip were statistically significant with the size of tumor and tumor grading $(p<0.05)$.
\end{abstract}

Conclusions: A statistical significance was found between the size of cervical nodes and the site of primary tumor of the oral tongue and lip. Herein, we recommended performing neck dissection in all cases of SCC of the base of the tongue, floor of the mouth, buccal mucosa, and retromolar trigone.

Keywords: Head and neck squamous cell carcinoma, Cervical node metastasis, Prognostic factors

\section{Background}

Head and neck squamous cell carcinoma (HNSCC) is one of the most common malignant tumors of the skin and oral cavity. Early diagnosis and treatment have a favorable prognosis. Regional metastasis of HNSCC is most likely to involve the cervical lymph node. Nodal status is one of prognostic factor and affects the survival rate of patients. Then, accurate staging of cervical lymph node is necessary. Histopathologic confirmation of metastatic node is the method to provide the final staging.

\footnotetext{
* Correspondence: pataranat@hotmail.com

'Division of Plastic and Reconstructive Surgery, Department of Surgery, Phramongkutklao Hospital and College of Medicine, 315 Ratchawithi Road, Thung Phayathai, Ratchathewi, Bangkok 10400, Thailand

Full list of author information is available at the end of the article
}

The investigations including computed tomography (CT) [1,2], magnetic resonance imaging (MRI) [3-5], positron emission tomography (PET) [6,7], and Doppler ultrasound [8] give more information to identify lymph nodes. Moreover, the biomarker study for prognostic factors such as matrix metalloproteinase-13 (MMP-13) [9], cyclooxygenase-2 (COX-2) expression [10], and gene expression (molecular analysis) marker [11] can predict occult metastasis but the accuracy is inconclusive.

The size of cervical lymph node is the guideline for operative procedures. Usually, cervical nodes larger than $10 \mathrm{~mm}$ are significant for nodal metastasis and the operation is a radical neck dissection. However, pathologically identified neck node metastasis occurs in $34 \%-51 \%$ of 
prophylactic neck dissections and $58 \%-69 \%$ in therapeutic neck dissections $[12,13]$.

At present, size criterion to diagnose occult malignant nodes is not reliable and presents a challenge for the surgeon to determine the extension of neck dissection. The association between nodal metastasis and size of cervical node is inconclusive and controversial.

The objective of this study was to identify prognostic factors of cervical node status including the site and size of primary tumors, presence of lymphovascular invasion, and size of cervical node for appropriate further treatment in HNSCC.

\section{Methods}

Approval of this study was obtained from the ethics committee of Phramongkutklao Hospital and College of Medicine. A 5-year retrospective review of patients with HNSCC in Phramongkutklao Hospital from 2009 to 2013 was conducted.

Inclusion criteria were patients with squamous cell carcinoma (SCC) of the lips, oral tongue, base of the tongue, floor of the mouth, buccal mucosa, alveolar, and retromolar trigone. The patients with non-SCC and cancer of the thyroid, pharynx, larynx, tonsils, and salivary glands were excluded.

The database included age, sex, tumor site, tumor size, tumor staging, histopathologic data of primary tumors and cervical nodes, presence of cervical lymph node metastasis, and treatment modality. Histopathologic data of primary tumors included differentiation of tumor, presence of lymphovascular invasion, and perineural invasion. The differentiation of tumor was dependent on features such as nuclear and cytoplasmic differentiation and degree of keratinization and was divided in a fourgrade system. Tumor differentiations were described as well differentiated, moderately differentiated, poorly differentiated, and undifferentiated.

The surgery of cervical neck nodes was divided into therapeutic neck dissection and prophylactic neck dissection. The therapeutic dissection was defined as neck dissection in patients with palpable cervical lymph nodes, or if the investigation showed cervical nodes larger than $8 \mathrm{~mm}$, it was defined as radical neck dissection or modified neck dissection. Prophylactic neck dissection was performed in patients with tumor size more than 2 $\mathrm{cm}$ and all cases of the base of the tongue. Prophylactic neck dissection was defined as modified neck dissection or supraomohyoid neck dissection.

All cervical nodes were reviewed including the size of the node, presence of metastasis, and extracapsular extension under a microscope by an experienced pathologist. Cervical nodes were divided into five groups: $1-3,4-6,7-9,10-30$, and $>30 \mathrm{~mm}$. Cervical nodes were compared in different sizes, extracapsular extension, and number of positive and negative nodes at each site of HNSCC.

\section{Statistic analysis}

Correlation between cervical nodes and tumors was analyzed. The univariate analysis of the independent variables was accomplished using Fisher's exact test or chi-square test. A $p$ value $<0.05$ was considered statistically significant.

\section{Results}

A total of 204 patients with HNSCC were recorded. Thirty-nine patients were excluded because of refused treatment, comorbidity, distant metastasis, and unavailability of medical records. The remaining 165 patients with HNSCC were reviewed. Overall, the mean age of the patients was 52.6 years (range, 23 to 89 years) (Table 1). Of these patients, $50.3 \%$ ranged in age from 45 to 60 years. There were 128 (77.58\%) male and 37 (22.42\%) female. Oral tongue was the most frequent primary site (50.91\%) and retromolar trigone was the least (3.03\%). Clinical stage I, stage II, stage III, and stage IV were diagnosed in $60.00 \%, 13.94 \%, 13.94 \%$, and $12.12 \%$, respectively. Tumor sizes were classified in five categories, $22.42 \%$ were smaller than $1 \mathrm{~cm}$ and $39.39 \%$ ranged in sizes between 1.1 and $2.0 \mathrm{~cm}$. Eighty-six patients (52.12\%) were categorized as having well differentiated, $49(29.70 \%)$ as moderately differentiated, $25(15.15 \%)$ as poorly differentiated, and $5(3.03 \%)$ as undifferentiated lesions.

Sixty-nine patients $(41.82 \%)$ underwent neck dissection and 1,472 nodes were found and reviewed (Table 2). The mean number of nodes was 21.33 nodes per patient. According to the histopathology reviewed, 87 patients (52.72\%) showed lymphovascular invasion (Table 3). Lymphovascular invasion of SCC of the oral tongue, base of the tongue, lip, floor of the mouth, buccal mucosa, and retromolar trigone were $42.85 \%, 66.67 \%, 58.06 \%$, $57.89 \%, 72.72 \%$, and $80.00 \%$, respectively. No statistical significance was found among lymphovascular invasion of primary tumor, clinical staging, and pathologic differentiation. A statistically significant association was found between the size of SCC of the oral tongue and lymphovascular invasion $(p<0.05)$ (Table 3$)$. Thirty patients $(69.77 \%)$ in therapeutic neck dissection and 15 patients $(57.69 \%)$ in prophylactic neck dissection showed lymphovascular invasion of the primary tumor.

Therapeutic neck dissections were performed in 43 patients $(62.32 \%)$ and prophylactic neck dissections were performed in 26 patients (37.68\%). Thirty-five patients $(81.40 \%)$ in the therapeutic neck dissections and 18 patients (69.23\%) in the prophylactic neck dissections showed metastasis of SCC to the cervical nodes. Fortythree patients underwent therapeutic neck dissection, 
Table 1 Demographic data of 165 patients of HNSCC

\begin{tabular}{|c|c|c|}
\hline Patient characteristics & Number & Percent \\
\hline \multicolumn{3}{|l|}{ Gender } \\
\hline Male & 128 & 77.58 \\
\hline Female & 37 & 22.42 \\
\hline \multicolumn{3}{|l|}{ Age } \\
\hline$<45$ years & 36 & 21.82 \\
\hline 45-60 years & 83 & 50.30 \\
\hline$>60$ years & 46 & 27.88 \\
\hline \multicolumn{3}{|l|}{ Site of SCC of the oral cavity } \\
\hline Oral tongue & 84 & 50.91 \\
\hline Base of the tongue & 15 & 9.09 \\
\hline Lip & 31 & 18.79 \\
\hline Floor of the mouth & 19 & 11.51 \\
\hline Buccal mucosa & 11 & 6.67 \\
\hline Retromolar trigone & 5 & 3.03 \\
\hline \multicolumn{3}{|l|}{ Clinical staging } \\
\hline Stage I & 99 & 60.00 \\
\hline Stage II & 23 & 13.94 \\
\hline Stage III & 23 & 13.94 \\
\hline Stage IV & 20 & 12.12 \\
\hline \multicolumn{3}{|l|}{ Tumor size } \\
\hline$\leq 1.0 \mathrm{~cm}$ & 37 & 22.42 \\
\hline $1.1-2.0 \mathrm{~cm}$ & 65 & 39.39 \\
\hline $2.1-3.0 \mathrm{~cm}$ & 23 & 13.94 \\
\hline $3.1-4.0 \mathrm{~cm}$ & 27 & 16.36 \\
\hline$>4.0 \mathrm{~cm}$ & 13 & 7.88 \\
\hline \multicolumn{3}{|l|}{ Pathologic differentiation } \\
\hline Well differentiated & 86 & 52.12 \\
\hline Moderately differentiated & 49 & 29.70 \\
\hline Poorly differentiated & 25 & 15.15 \\
\hline Undifferentiated & 5 & 3.03 \\
\hline
\end{tabular}

producing 956 nodes (average 22.23 nodes per patient) and 26 patients in prophylactic neck dissection group generated 516 nodes (average 19.85 nodes per patient) (Table 4). The most common size of cervical nodes was 1-3 mm. The mean size of nodes was $3.67 \mathrm{~mm}$ (range, 1-45 mm). Metastatic nodes totaled 289 nodes (19.63\%).

In all, 204 nodes (21.34\%) in the group of therapeutic neck dissection showed metastasis, and the mean size of metastatic nodes was $3.89 \mathrm{~mm}$ (range, 2-45 mm). Eighty-five nodes $(16.47 \%)$ in the prophylactic neck dissection group exhibited metastasis, and the mean size of metastatic nodes was $3.53 \mathrm{~mm}$ (range of metastasis nodes, 2-23 mm). In the therapeutic neck dissection group, a statistical significance was found between the size of cervical nodes and the site of primary tumor of the oral tongue, lip, and floor of the mouth $(p<0.05)$. In the prophylactic neck dissection group, a statistical significance was found between the size of cervical nodes and the site of primary tumor of the oral tongue, base of the tongue, and floor of the mouth $(p<0.05)$ (Table 4).

Among 289 metastatic nodes, 201 (69.55\%) showed extracapsular extension (Table 5). No statistical significance was found among extracapsular extension of cervical nodes and clinical staging, size of primary tumor, pathologic differentiation, and size of cervical nodes. According to each site of tumor, the size of cervical lymph node of SCC of the oral tongue was statistically significant with size of tumor and tumor grading $(p<0.05)$ (Table 6). The size of cervical lymph node of SCC of the lip was statistically significant with clinical staging and tumor grading $(p<0.05)$ (Table 7). No statistical significance was observed among SCC of the base of the tongue, floor of the mouth, buccal mucosa, and retromolar trigone and clinical staging, size of primary tumor, and pathologic differentiation (Tables 8, 9, 10, and 11).

Complications of neck dissection included wound infection, partial skin flap necrosis, and prolonged seroma. All complications were healed conservatively. Complications of neck dissection were found in six patients (13.95\%) in therapeutic neck dissection and three patients (11.54\%) in prophylactic neck dissection. No statistical significance was found among the complication rate between the therapeutic and prophylactic neck dissections.

\section{Discussion}

HNSCC is one of the most common cancers. Morbidity and mortality are related to regional and distant metastasis. The number and size of cervical node metastasis varies depending on the site, tumor differentiation, and stage of primary tumor. Cervical node metastasis is an important prognostic factor for HNSCC [12]. Kuperman et al. revealed the relationship between the risk of distant metastasis and tumor site, size, and nodal status [14]. The size of the cervical lymph node remains an important factor in the interpretation of a clinically suspicious lymph node metastasis; however, it remains controversial regarding the significance of the size of the cervical node.

The accuracy of staging depends on the status of the cervical node. Many methods have attempted to detect the node status but no gold standard exists, except the histopathologic examination. Some studies have reported that the size of the cervical node was an inaccurate predictor of nodal metastasis and could not be regarded as an accurate means of staging in patients with clinically negative nodes $[3,15]$. Neck dissection is both a therapeutic and staging procedure and has evolved to include various types with standardized level designations (I-VI) 
Table 2 Characteristics of squamous cell carcinoma depend on the site of primary tumor

\begin{tabular}{|c|c|c|c|c|c|c|}
\hline & \multicolumn{6}{|l|}{ Site of cancer } \\
\hline & Oral tongue & Base of the tongue & Lip & Floor of the mouth & Buccal mucosa & Retromolar trigone \\
\hline & $84(\%)$ & $15(\%)$ & $31(\%)$ & $19(\%)$ & $11(\%)$ & $5(\%)$ \\
\hline \multicolumn{7}{|l|}{ Clinical staging } \\
\hline Stage I & $68(80.95)$ & $3(20.00)$ & $19(61.29)$ & $4(21.05)$ & $5(45.45)$ & $0(0.00)$ \\
\hline Stage II & $3(3.57)$ & $5(33.33)$ & $6(19.35)$ & $6(31.58)$ & $2(18.18)$ & $1(20.00)$ \\
\hline Stage III & $8(9.52)$ & $3(20.00)$ & $3(9.68)$ & $5(26.32)$ & $2(18.18)$ & $2(40.00)$ \\
\hline Stage IV & $5(5.95)$ & $4(26.67)$ & $3(9.68)$ & $4(21.05)$ & $2(18.18)$ & $2(40.00)$ \\
\hline \multicolumn{7}{|l|}{ Tumor size } \\
\hline$\leq 1.0 \mathrm{~cm}$ & $22(26.19)$ & $1(6.67)$ & $11(35.48)$ & $2(10.53)$ & $1(9.09)$ & $0(0.00)$ \\
\hline $1.1-2.0 \mathrm{~cm}$ & $48(57.14)$ & $2(13.33)$ & $8(25.81)$ & $2(10.53)$ & $5(45.45)$ & $0(0.00)$ \\
\hline $2.1-3.0 \mathrm{~cm}$ & $4(4.76)$ & $4(26.67)$ & $7(22.58)$ & $6(31.58)$ & $2(18.18)$ & $0(0.00)$ \\
\hline $3.1-4.0 \mathrm{~cm}$ & $7(8.33)$ & $5(33.33)$ & $3(9.68)$ & $7(22.58)$ & $2(18.18)$ & $3(60.00)$ \\
\hline$>4.0 \mathrm{~cm}$ & $3(3.57)$ & $3(20.00)$ & $2(13.33)$ & $2(10.53)$ & $1(9.09)$ & $2(40.00)$ \\
\hline \multicolumn{7}{|l|}{ Pathologic differentiation } \\
\hline Well differentiated & $50(59.52)$ & $6(40.00)$ & $15(48.39)$ & $8(42.11)$ & $7(63.63)$ & $0(0.00)$ \\
\hline Moderately differentiated & $24(28.57)$ & $4(26.67)$ & $8(25.81)$ & $8(42.11)$ & $2(18.18)$ & $3(60.00)$ \\
\hline Poorly differentiated & $7(8.33)$ & $4(26.67)$ & $7(22.58)$ & $3(15.79)$ & $2(18.18)$ & $2(40.00)$ \\
\hline Undifferentiated & $3(3.57)$ & $1(6.67)$ & $1(3.23)$ & $0(0.00)$ & $0(0.00)$ & $0(0.00)$ \\
\hline \multicolumn{7}{|l|}{ Neck dissection } \\
\hline Therapeutic & $13(15.48)$ & $8(53.33)$ & $5(16.13)$ & $9(47.37)$ & $4(36.36)$ & $4(80.00)$ \\
\hline Prophylactic & $3(3.57)$ & $7(46.67)$ & $7(22.58)$ & $6(31.58)$ & $2(18.18)$ & $1(20.00)$ \\
\hline
\end{tabular}

for lymph node groups. Neck dissection is still a challenging treatment among patients with clinically negative nodes.

Ozer et al. suggested therapeutic neck dissection among patients with clinically negative nodes because pathologically positive nodes might be found in some patients [16]. Some reports have shown pathologically identified neck node metastasis occurred $34 \%-51 \%$ in prophylactic neck dissections [12,13]. In our study, $41.82 \%$ of HNSCC performed neck dissection. We found $69.23 \%$ of prophylactic neck dissections showed nodal metastasis with the size of the cervical lymph nodes varying from 2 to $23 \mathrm{~mm}$. Statistically significant associations were found between the size of cervical nodes and the site of primary tumor of the oral tongue, base of the tongue, and floor of the mouth. No statistical significance was observed between the size of cervical nodes and the site of primary tumor at the buccal mucosa, lip, and retromolar trigone.

Di et al. reported the significance of the size of lymph node and recurrence. The size of the cervical node metastasis is the key risk factor in determining the development of cervical recurrence. Patients presenting extracapsular nodal spread and invasion of non-lymphatic structures have a high risk of developing cervical recurrence [17].
Fine needle aspiration (FNA) is one of the methods to identify cervical node metastasis, but negative FNA could not exclude metastasis at the cervical node. Sentinel node biopsy of the cervical node is controversial. Broglie et al. reported the occult metastases detected by sentinel node biopsy in patients with early HNSCC [18]. However, the current knowledge of sentinel lymph node biopsy does not allow avoiding the indication of elective neck dissection in clinical practice. Sentinel lymph node biopsy cannot be considered the standard of care at this time [19].

Many studies have attempted to identify preoperative cervical node status, but most of them have been usually inconclusive. Matsubara et al. indicated that F-18 FDG $\mathrm{PET} / \mathrm{CT}$ was potentially useful in diagnosing preoperative nodal state [6]. Furthermore, a combined assessment of SUVmax with nodal size could be significant when identifying metastatic lymph nodes in HNSCC. Yamasaki et al. reported $80 \%$ false negative PET results in lymph nodes [7]. Kagawa et al. showed an increase in vascularity is a characteristic of Doppler ultrasound findings in small metastatic lymph nodes [8]. As the metastatic lymph node size increases, blood flow signals become scattered and the scattering index increases. Ding et al. demonstrated MRI diagnostic criteria of cervical lymph node metastasis include nodal size, central nodal necrosis, 
Table 3 Lymphovascular invasion of primary tumor

\begin{tabular}{|c|c|c|c|c|c|c|c|c|c|c|c|c|c|c|c|c|c|c|}
\hline & \multicolumn{18}{|c|}{ Lymphovascular invasion } \\
\hline & \multicolumn{2}{|c|}{$\begin{array}{l}\text { Oral tongue } \\
(84)\end{array}$} & \multirow[t]{2}{*}{$p$ value $^{a}$} & \multicolumn{2}{|c|}{$\begin{array}{l}\text { Base of the tongue } \\
\text { (15) }\end{array}$} & \multirow[t]{2}{*}{$p$ value $^{a}$} & \multicolumn{2}{|c|}{$\begin{array}{l}\text { Lip } \\
(31) \\
\end{array}$} & \multirow[t]{2}{*}{$p$ value $^{a}$} & \multicolumn{2}{|c|}{$\begin{array}{l}\text { Floor of the mouth } \\
\text { (19) }\end{array}$} & \multirow[t]{2}{*}{$p$ value $^{a}$} & \multicolumn{2}{|c|}{$\begin{array}{l}\text { Buccal mucosa } \\
\text { (11) }\end{array}$} & \multirow[t]{2}{*}{$p$ value $^{a}$} & \multicolumn{2}{|c|}{$\begin{array}{l}\text { Retromolar trigone } \\
(5)\end{array}$} & \multirow[t]{2}{*}{$p$ value $^{a}$} \\
\hline & + & - & & + & - & & + & - & & + & - & & + & - & & + & - & \\
\hline Clinical staging & & & 0.127 & & & 0.825 & & & 0.595 & & & 0.898 & & & 0.547 & & & NA \\
\hline Stage I & 25 & 43 & & 2 & 1 & & 12 & 7 & & 2 & 2 & & 2 & 3 & & 0 & 0 & \\
\hline Stage ॥ & 2 & 1 & & 2 & 2 & & 2 & 4 & & 4 & 2 & & 1 & 1 & & 1 & 0 & \\
\hline Stage III & 6 & 2 & & 2 & 1 & & 2 & 1 & & 3 & 2 & & 2 & 0 & & 2 & 0 & \\
\hline Stage IV & 3 & 2 & & 4 & 1 & & 2 & 1 & & 3 & 1 & & 1 & 1 & & 1 & 1 & \\
\hline Tumor size & & & 0.012 & & & 0.800 & & & 0.266 & & & 0.546 & & & 0.662 & & & NA \\
\hline$\leq 1.0 \mathrm{~cm}$ & 3 & 19 & & 0 & 1 & & 6 & 5 & & 0 & 1 & & 1 & 0 & & 0 & 0 & \\
\hline $1.1-2.0 \mathrm{~cm}$ & 23 & 25 & & 1 & 1 & & 7 & 1 & & 1 & 1 & & 3 & 2 & & 0 & 0 & \\
\hline $2.1-3.0 \mathrm{~cm}$ & 3 & 1 & & 3 & 1 & & 2 & 4 & & 4 & 2 & & 2 & 1 & & 0 & 0 & \\
\hline $3.1-4.0 \mathrm{~cm}$ & 5 & 2 & & 4 & 1 & & 2 & 1 & & 4 & 3 & & 3 & 0 & & 2 & 1 & \\
\hline$>4.0 \mathrm{~cm}$ & 2 & 1 & & 2 & 1 & & 1 & 2 & & 2 & 0 & & 1 & 1 & & 2 & 0 & \\
\hline Pathologic differentiation & & & 0.339 & & & 0.804 & & & 0.843 & & & 0.564 & & & NA & & & NA \\
\hline Well differentiated & 22 & 28 & & 3 & 3 & & 9 & 5 & & 6 & 2 & & 5 & 2 & & 0 & 0 & \\
\hline Moderately differentiated & 8 & 16 & & 2 & 2 & & 4 & 5 & & 4 & 4 & & 2 & 0 & & 2 & 0 & \\
\hline Poorly differentiated & 5 & 2 & & 1 & 3 & & 4 & 3 & & 1 & 2 & & 1 & 1 & & 0 & 0 & \\
\hline Undifferentiated & 1 & 2 & & 1 & 0 & & 1 & 0 & & 0 & 0 & & 0 & 0 & & 2 & 1 & \\
\hline Neck dissection & & & 1.000 & & & 0.608 & & & 1.000 & & & 1.000 & & & 1.000 & & & NA \\
\hline Therapeutic & 9 & 4 & & 6 & 2 & & 3 & 2 & & 6 & 3 & & 3 & 1 & & 3 & 1 & \\
\hline Prophylactic & 2 & 1 & & 4 & 3 & & 3 & 4 & & 4 & 2 & & 1 & 1 & & 1 & 0 & \\
\hline
\end{tabular}

${ }^{a}$ Chi-square test or Fisher's exact test.

+ primary tumor had lymphovascular invasion, - primary tumor did not have lymphovascular invasion. 


\section{Table 4 Size of neck node and metastasis}

\begin{tabular}{|c|c|c|c|c|c|c|c|c|c|c|c|c|c|c|c|c|c|c|}
\hline \multirow[t]{2}{*}{ Size of neck node } & \multicolumn{2}{|c|}{$\begin{array}{l}\text { Oral tongue } \\
(410)\end{array}$} & \multirow[t]{2}{*}{$p$ value $^{\mathrm{a}}$} & \multicolumn{2}{|c|}{$\begin{array}{l}\text { Base of the tongue } \\
\text { (319) }\end{array}$} & \multirow[t]{2}{*}{$p$ value $^{a}$} & \multicolumn{2}{|c|}{$\begin{array}{l}\text { Lip } \\
(238)\end{array}$} & \multirow[t]{2}{*}{$p$ value $^{a}$} & \multicolumn{2}{|c|}{$\begin{array}{l}\text { Floor of the mouth } \\
\text { (231) }\end{array}$} & \multirow[t]{2}{*}{$p$ value $^{a}$} & \multicolumn{2}{|c|}{$\begin{array}{l}\text { Buccal mucosa } \\
\text { (159) }\end{array}$} & \multirow[t]{2}{*}{$p$ value $^{a}$} & \multicolumn{2}{|c|}{$\begin{array}{l}\text { Retromolar trigone } \\
\text { (115) }\end{array}$} & \multirow[t]{2}{*}{$p$ value $^{a}$} \\
\hline & + & - & & + & - & & + & $\overline{-}$ & & + & - & & + & - & & + & - & \\
\hline Therapeutic neck dissection & & & $<0.001$ & & & 0.072 & & & 0.012 & & & 0.002 & & & 0.231 & & & 0.682 \\
\hline $1-3 \mathrm{~mm}$ & 7 & 78 & & 5 & 35 & & 3 & 27 & & 2 & 40 & & 2 & 18 & & 2 & 12 & \\
\hline $4-6 \mathrm{~mm}$ & 10 & 61 & & 7 & 42 & & 5 & 29 & & 6 & 25 & & 5 & 14 & & 3 & 7 & \\
\hline 7-9 mm & 11 & 31 & & 7 & 39 & & 6 & 38 & & 9 & 28 & & 7 & 21 & & 5 & 10 & \\
\hline $10-30 \mathrm{~mm}$ & 31 & 53 & & 16 & 36 & & 18 & 33 & & 13 & 19 & & 9 & 21 & & 12 & 33 & \\
\hline$>30 \mathrm{~mm}$ & 1 & 1 & & 0 & 0 & & 0 & 0 & & 0 & 0 & & 2 & 1 & & 0 & 0 & \\
\hline Prophylactic neck dissection & & & 0.006 & & & 0.013 & & & 0.150 & & & 0.007 & & & 0.628 & & & 0.898 \\
\hline $1-3 \mathrm{~mm}$ & 2 & 34 & & 2 & 26 & & 1 & 12 & & 2 & 28 & & 1 & 11 & & 1 & 4 & \\
\hline $4-6 \mathrm{~mm}$ & 4 & 28 & & 4 & 32 & & 2 & 14 & & 3 & 21 & & 2 & 14 & & 1 & 4 & \\
\hline 7-9 mm & 4 & 30 & & 5 & 35 & & 2 & 21 & & 3 & 17 & & 3 & 17 & & 2 & 7 & \\
\hline $10-30 \mathrm{~mm}$ & 9 & 15 & & 10 & 18 & & 8 & 19 & & 7 & 8 & & 3 & 8 & & 4 & 8 & \\
\hline$>30 \mathrm{~mm}$ & 0 & 0 & & 0 & 0 & & 0 & 0 & & 0 & 0 & & 0 & 0 & & 0 & 0 & \\
\hline
\end{tabular}

${ }^{a}$ Chi-square test or Fisher's exact test.

+ metastasis of SCC to cervical lymph node, - no metastasis of SCC to cervical lymph node. 
Table 5 Analysis of extracapsular extension of metastasis cervical node in each site of SCC

\begin{tabular}{|c|c|c|c|c|c|c|c|c|c|c|c|c|c|c|c|c|c|c|}
\hline & \multicolumn{18}{|c|}{ Extracapsular extension } \\
\hline & \multicolumn{2}{|c|}{$\begin{array}{l}\text { Oral tongue } \\
(79)\end{array}$} & \multirow[t]{2}{*}{$p$ value $^{\mathrm{a}}$} & \multicolumn{2}{|c|}{$\begin{array}{l}\text { Base of the tongue } \\
\text { (56) }\end{array}$} & \multirow[t]{2}{*}{$p$ value $^{a}$} & \multicolumn{2}{|c|}{$\begin{array}{l}\text { Lip } \\
\text { (45) }\end{array}$} & \multirow[t]{2}{*}{$p$ value $^{a}$} & \multicolumn{2}{|c|}{$\begin{array}{l}\text { Floor of the mouth } \\
\text { (45) }\end{array}$} & \multirow[t]{2}{*}{$p$ value $^{a}$} & \multicolumn{2}{|c|}{$\begin{array}{l}\text { Buccal mucosa } \\
\text { (34) }\end{array}$} & \multirow[t]{2}{*}{$p$ value $^{a}$} & \multicolumn{2}{|c|}{$\begin{array}{l}\text { Retromolar trigone } \\
\text { (30) }\end{array}$} & \multirow[t]{2}{*}{$p$ value } \\
\hline & + & - & & + & - & & + & - & & + & - & & + & - & & + & - & \\
\hline Clinical staging & & & 0.465 & & & 0.518 & & & 0.195 & & & 0.255 & & & 0.891 & & & 0.634 \\
\hline Stage I & 0 & 0 & & 5 & 5 & & 0 & 0 & & 0 & 0 & & 0 & 0 & & 0 & 0 & \\
\hline Stage II & 6 & 4 & & 11 & 6 & & 9 & 8 & & 8 & 6 & & 3 & 2 & & 2 & 2 & \\
\hline Stage III & 25 & 11 & & 9 & 5 & & 12 & 3 & & 12 & 3 & & 10 & 4 & & 9 & 5 & \\
\hline Stage IV & 26 & 7 & & 12 & 3 & & 10 & 3 & & 13 & 3 & & 10 & 5 & & 9 & 3 & \\
\hline Tumor size & & & 0.121 & & & 0.318 & & & 0.433 & & & 0.865 & & & 0.898 & & & 1.000 \\
\hline$\leq 1.0 \mathrm{~cm}$ & 0 & 0 & & 3 & 4 & & 0 & 0 & & 0 & 0 & & 0 & 0 & & 0 & 0 & \\
\hline $1.1-2.0 \mathrm{~cm}$ & 3 & 4 & & 3 & 4 & & 0 & 0 & & 0 & 0 & & 0 & 0 & & 0 & 0 & \\
\hline $2.1-3.0 \mathrm{~cm}$ & 11 & 7 & & 11 & 3 & & 13 & 8 & & 11 & 5 & & 7 & 4 & & 0 & 0 & \\
\hline $3.1-4.0 \mathrm{~cm}$ & 19 & 5 & & 13 & 5 & & 8 & 4 & & 15 & 5 & & 8 & 4 & & 11 & 6 & \\
\hline$>4.0 \mathrm{~cm}$ & 24 & 6 & & 7 & 3 & & 10 & 2 & & 7 & 2 & & 8 & 3 & & 9 & 4 & \\
\hline Pathologic differentiation & & & 0.874 & & & 0.447 & & & 0.718 & & & 0.474 & & & 0.654 & & & 0.705 \\
\hline Well differentiated & 21 & 7 & & 10 & 9 & & 7 & 5 & & 10 & 6 & & 7 & 3 & & 8 & 5 & \\
\hline Moderately differentiated & 25 & 9 & & 12 & 5 & & 8 & 2 & & 15 & 4 & & 7 & 2 & & 0 & 0 & \\
\hline Poorly differentiated & 6 & 3 & & 11 & 3 & & 10 & 5 & & 8 & 2 & & 9 & 6 & & 12 & 5 & \\
\hline Undifferentiated & 5 & 3 & & 4 & 2 & & 6 & 2 & & 0 & 0 & & 0 & 0 & & 0 & 0 & \\
\hline Size of neck node & & & 0.506 & & & 0.793 & & & 0.849 & & & 0.968 & & & 0.824 & & & 0.994 \\
\hline $1-3 \mathrm{~mm}$ & 5 & 4 & & 4 & 3 & & 3 & 1 & & 3 & 1 & & 2 & 1 & & 2 & 1 & \\
\hline $4-6 \mathrm{~mm}$ & 9 & 5 & & 7 & 4 & & 4 & 3 & & 6 & 3 & & 4 & 3 & & 3 & 1 & \\
\hline 7-9 mm & 11 & 4 & & 9 & 3 & & 6 & 2 & & 9 & 3 & & 6 & 4 & & 5 & 2 & \\
\hline $10-30 \mathrm{~mm}$ & 31 & 9 & & 17 & 9 & & 18 & 8 & & 15 & 5 & & 9 & 3 & & 10 & 6 & \\
\hline$>30 \mathrm{~mm}$ & 1 & 0 & & 0 & 0 & & 0 & 0 & & 0 & 0 & & 2 & 0 & & 0 & 0 & \\
\hline
\end{tabular}

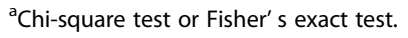

+ extracapsular extension of metastasis cervical node, - no extracapsular extension of metastasis cervical node. 
Table 6 Size of cervical lymph node and metastasis in oral tongue squamous cell carcinoma

\begin{tabular}{|c|c|c|c|c|c|c|c|c|c|c|c|c|c|c|c|}
\hline & \multicolumn{15}{|c|}{ Size of cervical node } \\
\hline & \multicolumn{2}{|c|}{$1-3 \mathrm{~mm}$} & \multirow[t]{3}{*}{$p$ value $^{a}$} & \multicolumn{2}{|c|}{$4-6 \mathrm{~mm}$} & \multirow[t]{3}{*}{$p$ value $^{a}$} & \multicolumn{2}{|c|}{ 7-9 mm } & \multirow[t]{3}{*}{$p$ value $^{a}$} & \multicolumn{2}{|c|}{$10-30 \mathrm{~mm}$} & \multirow[t]{3}{*}{$p$ value $^{a}$} & \multicolumn{2}{|c|}{$>30 \mathrm{~mm}$} & \multirow[t]{3}{*}{$p$ value } \\
\hline & + & - & & + & - & & + & - & & + & - & & + & - & \\
\hline & 9 & 112 & & 14 & 89 & & 15 & 61 & & 40 & 68 & & 1 & 1 & \\
\hline Clinical staging & & & 0.354 & & & 0.794 & & & 0.576 & & & 0.320 & & & $\mathrm{NA}$ \\
\hline Stage I & 0 & 0 & & 0 & 0 & & 0 & 0 & & 0 & 0 & & 0 & 0 & \\
\hline Stage II & 0 & 9 & & 2 & 8 & & 4 & 11 & & 4 & 12 & & 0 & 0 & \\
\hline Stage III & 3 & 54 & & 6 & 44 & & 5 & 29 & & 22 & 28 & & 0 & 0 & \\
\hline Stage IV & 6 & 49 & & 6 & 37 & & 6 & 21 & & 14 & 28 & & 1 & 1 & \\
\hline Tumor size & & & 0.033 & & & 0.467 & & & 0.915 & & & 0.140 & & & NA \\
\hline$\leq 1.0 \mathrm{~cm}$ & 0 & 0 & & 0 & 0 & & 0 & 0 & & 0 & 0 & & 0 & 0 & \\
\hline $1.1-2.0 \mathrm{~cm}$ & 1 & 61 & & 3 & 10 & & 3 & 9 & & 0 & 0 & & 0 & 0 & \\
\hline $2.1-3.0 \mathrm{~cm}$ & 3 & 15 & & 4 & 18 & & 6 & 24 & & 5 & 15 & & 0 & 0 & \\
\hline $3.1-4.0 \mathrm{~cm}$ & 2 & 24 & & 3 & 36 & & 3 & 17 & & 16 & 33 & & 0 & 0 & \\
\hline$>4.0 \mathrm{~cm}$ & 3 & 12 & & 4 & 25 & & 3 & 11 & & 19 & 20 & & 1 & 1 & \\
\hline Pathologic differentiation & & & 0.044 & & & 0.711 & & & 0.278 & & & 0.028 & & & NA \\
\hline Well differentiated & 2 & 58 & & 4 & 35 & & 4 & 29 & & 18 & 24 & & 0 & 0 & \\
\hline Moderately differentiated & 2 & 32 & & 6 & 27 & & 7 & 14 & & 19 & 22 & & 0 & 0 & \\
\hline Poorly differentiated & 4 & 13 & & 2 & 18 & & 2 & 11 & & 1 & 12 & & 0 & 0 & \\
\hline Undifferentiated & 1 & 9 & & 2 & 9 & & 2 & 7 & & 2 & 10 & & 1 & 1 & \\
\hline
\end{tabular}

${ }^{a}$ Chi-square test or Fisher' $s$ exact test.

+ metastasis of SCC to cervical node, - no metastasis of SCC to cervical node.

Table 7 Size of lymph node and metastasis in squamous cell carcinoma of lip

\begin{tabular}{|c|c|c|c|c|c|c|c|c|c|c|c|c|c|c|c|}
\hline & \multicolumn{15}{|c|}{ Size of cervical node } \\
\hline & \multicolumn{2}{|c|}{$1-3 \mathrm{~mm}$} & \multirow[t]{3}{*}{$p$ value $^{a}$} & \multicolumn{2}{|c|}{$4-6 \mathrm{~mm}$} & \multirow[t]{2}{*}{$p$ value $^{a}$} & \multicolumn{2}{|c|}{ 7-9 mm } & \multirow[t]{2}{*}{$p$ value $^{a}$} & \multicolumn{2}{|c|}{$10-30 \mathrm{~mm}$} & \multirow[t]{3}{*}{$p$ value $^{a}$} & \multicolumn{2}{|c|}{$>30 \mathrm{~mm}$} & \multirow[t]{3}{*}{$p$ value $^{a}$} \\
\hline & + & - & & + & - & & + & - & & + & - & & + & - & \\
\hline & 4 & 39 & & 7 & 43 & & 8 & 59 & & 26 & 52 & & 0 & 0 & \\
\hline Clinical staging & & & 0.344 & & & 0.492 & & & 0.238 & & & 0.015 & & & NA \\
\hline Stage I & 0 & 0 & & 0 & 0 & & 0 & 0 & & 0 & 0 & & 0 & 0 & \\
\hline Stage II & 0 & 14 & & 1 & 16 & & 1 & 17 & & 15 & 15 & & 0 & 0 & \\
\hline Stage III & 2 & 12 & & 3 & 14 & & 2 & 23 & & 8 & 16 & & 0 & 0 & \\
\hline Stage IV & 2 & 13 & & 3 & 13 & & 5 & 19 & & 3 & 21 & & 0 & 0 & \\
\hline Tumor size & & & 0.804 & & & 0.832 & & & 0.832 & & & 0.081 & & & NA \\
\hline$\leq 1.0 \mathrm{~cm}$ & 0 & 0 & & 0 & 0 & & 0 & 0 & & 0 & 0 & & & & \\
\hline $1.1-2.0 \mathrm{~cm}$ & 0 & 0 & & 0 & 0 & & 0 & 0 & & 0 & 0 & & 0 & 0 & \\
\hline $2.1-3.0 \mathrm{~cm}$ & 2 & 14 & & 3 & 17 & & 3 & 18 & & 13 & 15 & & 0 & 0 & \\
\hline $3.1-4.0 \mathrm{~cm}$ & 1 & 16 & & 2 & 17 & & 2 & 21 & & 7 & 19 & & 0 & 0 & \\
\hline$>4.0 \mathrm{~cm}$ & 1 & 9 & & 2 & 9 & & 3 & 20 & & 6 & 24 & & 0 & 0 & \\
\hline Pathologic differentiation & & & 0.776 & & & 0.709 & & & 0.907 & & & $<0.001$ & & & NA \\
\hline Well differentiated & 1 & 14 & & 2 & 18 & & 3 & 18 & & 6 & 27 & & 0 & 0 & \\
\hline Moderately differentiated & 2 & 11 & & 2 & 14 & & 2 & 18 & & 4 & 19 & & 0 & 0 & \\
\hline Poorly differentiated & 1 & 10 & & 2 & 9 & & 2 & 11 & & 10 & 4 & & 0 & 0 & \\
\hline Undifferentiated & 0 & 4 & & 1 & 2 & & 1 & 12 & & 6 & 2 & & 0 & 0 & \\
\hline
\end{tabular}

${ }^{\mathrm{a}} \mathrm{Chi}$-square test or Fisher's exact test.

+ metastasis of SCC to cervical node, - no metastasis of SCC to cervical node. 
Table 8 Size of lymph node and metastasis of squamous cell carcinoma of the base of the tongue

\begin{tabular}{|c|c|c|c|c|c|c|c|c|c|c|c|c|c|c|c|}
\hline & \multicolumn{15}{|c|}{ Size of cervical node } \\
\hline & \multicolumn{2}{|c|}{$1-3 \mathrm{~mm}$} & \multirow[t]{3}{*}{$p$ value $^{a}$} & \multicolumn{2}{|c|}{$4-6 \mathrm{~mm}$} & \multirow[t]{3}{*}{$p$ value $^{a}$} & \multicolumn{2}{|c|}{$7-9 \mathrm{~mm}$} & \multirow[t]{3}{*}{$p$ value $^{a}$} & \multicolumn{2}{|c|}{$10-30 \mathrm{~mm}$} & \multirow[t]{3}{*}{$p$ value $^{a}$} & \multicolumn{2}{|c|}{$>30 \mathrm{~mm}$} & \multirow[t]{3}{*}{$p$ value } \\
\hline & + & - & & + & - & & + & - & & + & - & & + & - & \\
\hline & 7 & 61 & & 11 & 74 & & 12 & 74 & & 26 & 54 & & 0 & 0 & \\
\hline Clinical staging & & & 0.280 & & & 0.976 & & & 0.602 & & & 0.999 & & & NA \\
\hline Stage I & 1 & 11 & & 2 & 11 & & 3 & 9 & & 4 & 8 & & 0 & 0 & \\
\hline Stage II & 1 & 23 & & 3 & 25 & & 4 & 28 & & 9 & 19 & & 0 & 0 & \\
\hline Stage III & 2 & 18 & & 3 & 19 & & 2 & 21 & & 7 & 14 & & 0 & 0 & \\
\hline Stage IV & 3 & 9 & & 3 & 19 & & 3 & 16 & & 6 & 13 & & 0 & 0 & \\
\hline Tumor size & & & 0.441 & & & 0.413 & & & 0.756 & & & 0.161 & & & NA \\
\hline$\leq 1.0 \mathrm{~cm}$ & 0 & 8 & & 1 & 5 & & 1 & 7 & & 5 & 6 & & & & \\
\hline $1.1-2.0 \mathrm{~cm}$ & 1 & 14 & & 2 & 5 & & 2 & 5 & & 2 & 6 & & 0 & 0 & \\
\hline $2.1-3.0 \mathrm{~cm}$ & 1 & 17 & & 2 & 27 & & 2 & 21 & & 9 & 12 & & 0 & 0 & \\
\hline $3.1-4.0 \mathrm{~cm}$ & 3 & 15 & & 3 & 27 & & 4 & 21 & & 8 & 13 & & 0 & 0 & \\
\hline$>4.0 \mathrm{~cm}$ & 2 & 7 & & 3 & 10 & & 3 & 20 & & 2 & 17 & & 0 & 0 & \\
\hline Pathologic differentiation & & & 0.546 & & & 0.948 & & & 0.765 & & & 0.587 & & & NA \\
\hline Well differentiated & 4 & 21 & & 5 & 30 & & 4 & 34 & & 6 & 18 & & 0 & 0 & \\
\hline Moderately differentiated & 2 & 17 & & 3 & 22 & & 3 & 20 & & 9 & 18 & & 0 & 0 & \\
\hline Poorly differentiated & 1 & 14 & & 2 & 13 & & 3 & 12 & & 8 & 10 & & 0 & 0 & \\
\hline Undifferentiated & 0 & 9 & & 1 & 9 & & 2 & 8 & & 3 & 8 & & 0 & 0 & \\
\hline
\end{tabular}

${ }^{a}$ Chi-square test or Fisher' s exact test.

+ metastasis of SCC to cervical node, - no metastasis of SCC to cervical node.

Table 9 Size of lymph node and metastasis of squamous cell carcinoma of the floor of the mouth

\begin{tabular}{|c|c|c|c|c|c|c|c|c|c|c|c|c|c|c|c|}
\hline & \multicolumn{15}{|c|}{ Size of cervical node } \\
\hline & \multicolumn{2}{|c|}{$1-3 \mathrm{~mm}$} & \multirow[t]{3}{*}{$p$ value $^{a}$} & \multicolumn{2}{|c|}{$4-6 \mathrm{~mm}$} & \multirow[t]{3}{*}{$p$ value $^{a}$} & \multicolumn{2}{|c|}{ 7-9 mm } & \multirow[t]{3}{*}{$p$ value $^{a}$} & \multicolumn{2}{|c|}{$10-30 \mathrm{~mm}$} & \multirow[t]{3}{*}{$p$ value $^{a}$} & \multicolumn{2}{|c|}{$>30 \mathrm{~mm}$} & \multirow[t]{3}{*}{$p$ value $^{a}$} \\
\hline & + & - & & + & - & & + & - & & + & - & & + & - & \\
\hline & 4 & 68 & & 9 & 46 & & 12 & 45 & & 20 & 27 & & & & \\
\hline Clinical staging & & & 0.591 & & & 0.598 & & & 0.437 & & & 0.946 & & & NA \\
\hline Stage I & 0 & 0 & & 0 & 0 & & 0 & 0 & & 0 & 0 & & 0 & 0 & \\
\hline Stage II & 1 & 23 & & 2 & 16 & & 5 & 13 & & 6 & 7 & & 0 & 0 & \\
\hline Stage III & 1 & 27 & & 3 & 17 & & 5 & 16 & & 6 & 9 & & 0 & 0 & \\
\hline Stage IV & 2 & 18 & & 4 & 13 & & 2 & 16 & & 8 & 11 & & 0 & 0 & \\
\hline Tumor size & & & 0.859 & & & 0.913 & & & 0.347 & & & 0.871 & & & NA \\
\hline$\leq 1.0 \mathrm{~cm}$ & 0 & 0 & & 0 & 0 & & 0 & 0 & & 0 & 0 & & & & \\
\hline $1.1-2.0 \mathrm{~cm}$ & 0 & 0 & & 0 & 0 & & 0 & 0 & & 0 & 0 & & 0 & 0 & \\
\hline $2.1-3.0 \mathrm{~cm}$ & 1 & 19 & & 3 & 17 & & 5 & 18 & & 7 & 8 & & 0 & 0 & \\
\hline $3.1-4.0 \mathrm{~cm}$ & 1 & 24 & & 4 & 17 & & 6 & 15 & & 9 & 12 & & 0 & 0 & \\
\hline$>4.0 \mathrm{~cm}$ & 2 & 25 & & 2 & 12 & & 1 & 12 & & 4 & 7 & & 0 & 0 & \\
\hline Pathologic differentiation & & & 0.933 & & & 0.803 & & & 0.868 & & & 0.186 & & & NA \\
\hline Well differentiated & 2 & 31 & & 4 & 21 & & 6 & 19 & & 4 & 10 & & 0 & 0 & \\
\hline Moderately differentiated & 1 & 23 & & 2 & 14 & & 4 & 16 & & 12 & 9 & & 0 & 0 & \\
\hline Poorly differentiated & 1 & 14 & & 3 & 11 & & 2 & 10 & & 4 & 8 & & 0 & 0 & \\
\hline Undifferentiated & 0 & 0 & & 0 & 0 & & 0 & 0 & & 0 & 0 & & 0 & 0 & \\
\hline
\end{tabular}

${ }^{a}$ Chi-square test or Fisher's exact test.

+ metastasis of SCC to cervical node, - no metastasis of SCC to cervical node. 
Table 10 Size of lymph node and metastasis in squamous cell carcinoma of buccal mucosa

\begin{tabular}{|c|c|c|c|c|c|c|c|c|c|c|c|c|c|c|c|}
\hline & \multicolumn{15}{|c|}{ Size of cervical node } \\
\hline & \multicolumn{2}{|c|}{$1-3 \mathrm{~mm}$} & \multirow[t]{3}{*}{$p$ value $^{a}$} & \multicolumn{2}{|c|}{$4-6 \mathrm{~mm}$} & \multirow[t]{3}{*}{$p$ value $^{a}$} & \multicolumn{2}{|c|}{$7-9 \mathrm{~mm}$} & \multirow[t]{3}{*}{$p$ value $^{a}$} & \multicolumn{2}{|c|}{$10-30 \mathrm{~mm}$} & \multirow[t]{3}{*}{$p$ value $^{a}$} & \multicolumn{2}{|c|}{$>30 \mathrm{~mm}$} & \multirow[t]{3}{*}{$p$ value $^{a}$} \\
\hline & + & - & & + & - & & + & - & & + & - & & + & - & \\
\hline & 3 & 29 & & 7 & 28 & & 10 & 38 & & 12 & 29 & & 2 & 1 & \\
\hline Clinical staging & & & 0.468 & & & 0.525 & & & 0.686 & & & 0.553 & & & NA \\
\hline Stage I & 0 & 0 & & 0 & 0 & & 0 & 0 & & 0 & 0 & & 0 & 0 & \\
\hline Stage II & 0 & 7 & & 1 & 9 & & 2 & 13 & & 2 & 9 & & 0 & 0 & \\
\hline Stage III & 1 & 12 & & 4 & 10 & & 4 & 13 & & 5 & 12 & & 0 & 0 & \\
\hline Stage IV & 2 & 10 & & 2 & 9 & & 4 & 12 & & 5 & 8 & & 2 & 1 & \\
\hline Tumor size & & & 0.392 & & & 0.870 & & & 0.413 & & & 0.686 & & & NA \\
\hline$\leq 1.0 \mathrm{~cm}$ & 0 & 0 & & 0 & 0 & & 0 & 0 & & 0 & 0 & & 0 & 0 & \\
\hline $1.1-2.0 \mathrm{~cm}$ & 0 & 0 & & 0 & 0 & & 0 & 0 & & 0 & 0 & & 0 & 0 & \\
\hline $2.1-3.0 \mathrm{~cm}$ & 0 & 8 & & 2 & 7 & & 2 & 14 & & 7 & 13 & & 0 & 0 & \\
\hline $3.1-4.0 \mathrm{~cm}$ & 1 & 12 & & 3 & 10 & & 5 & 11 & & 3 & 8 & & 0 & 0 & \\
\hline$>4.0 \mathrm{~cm}$ & 2 & 9 & & 2 & 11 & & 3 & 13 & & 2 & 8 & & 2 & 1 & \\
\hline Pathologic differentiation & & & 0.988 & & & 0.975 & & & 0.603 & & & 0.240 & & & NA \\
\hline Well differentiated & 1 & 11 & & 3 & 12 & & 2 & 14 & & 4 & 12 & & 0 & 0 & \\
\hline Moderately differentiated & 1 & 9 & & 2 & 9 & & 4 & 12 & & 2 & 10 & & 0 & 0 & \\
\hline Poorly differentiated & 1 & 9 & & 2 & 7 & & 4 & 12 & & 6 & 7 & & 2 & 1 & \\
\hline Undifferentiated & 0 & 0 & & 0 & 0 & & 0 & 0 & & 0 & 0 & & 0 & 0 & \\
\hline
\end{tabular}

${ }^{a}$ Chi-square test or Fisher's exact test.

+ metastasis of SCC to cervical node, - no metastasis of SCC to cervical node.

Table 11 Size of lymph node and metastasis in squamous cell carcinoma of retromolar trigone

\begin{tabular}{|c|c|c|c|c|c|c|c|c|c|c|c|c|c|c|c|}
\hline & \multicolumn{15}{|c|}{ Size of cervical node } \\
\hline & \multicolumn{2}{|c|}{$1-3 \mathrm{~mm}$} & \multirow[t]{3}{*}{$p$ value $^{a}$} & \multicolumn{2}{|c|}{$4-6 \mathrm{~mm}$} & \multirow[t]{3}{*}{$p$ value $^{a}$} & \multicolumn{2}{|c|}{ 7-9 mm } & \multirow[t]{3}{*}{$p$ value $^{a}$} & \multicolumn{2}{|c|}{$10-30 \mathrm{~mm}$} & \multirow[t]{3}{*}{$p$ value $^{a}$} & \multicolumn{2}{|c|}{$>30 \mathrm{~mm}$} & \multirow[t]{3}{*}{$p$ value $^{a}$} \\
\hline & + & - & & + & - & & + & - & & + & - & & + & - & \\
\hline & 3 & 16 & & 4 & 11 & & 7 & 17 & & 16 & 41 & & & & \\
\hline Clinical staging & & & 0.484 & & & 0.880 & & & 0.931 & & & 0.231 & & & NA \\
\hline Stage I & 0 & 0 & & 0 & 0 & & 0 & 0 & & 0 & 0 & & 0 & 0 & \\
\hline Stage II & 0 & 5 & & 1 & 3 & & 2 & 5 & & 1 & 11 & & 0 & 0 & \\
\hline Stage III & 1 & 5 & & 1 & 4 & & 3 & 6 & & 9 & 18 & & 0 & 0 & \\
\hline Stage IV & 2 & 6 & & 2 & 4 & & 2 & 6 & & 6 & 12 & & 0 & 0 & \\
\hline Tumor size & & & 0.582 & & & 1.000 & & & 0.659 & & & 0.082 & & & NA \\
\hline$\leq 1.0 \mathrm{~cm}$ & 0 & 0 & & 0 & 0 & & 0 & 0 & & 0 & 0 & & 0 & 0 & \\
\hline $1.1-2.0 \mathrm{~cm}$ & 0 & 0 & & 0 & 0 & & 0 & 0 & & 0 & 0 & & 0 & 0 & \\
\hline $2.1-3.0 \mathrm{~cm}$ & 0 & 0 & & 0 & 0 & & 0 & 0 & & 0 & 0 & & 0 & 0 & \\
\hline $3.1-4.0 \mathrm{~cm}$ & 1 & 9 & & 2 & 6 & & 3 & 10 & & 11 & 17 & & 0 & 0 & \\
\hline$>4.0 \mathrm{~cm}$ & 2 & 7 & & 2 & 5 & & 4 & 7 & & 5 & 24 & & 0 & 0 & \\
\hline Pathologic differentiation & & & 0.546 & & & 0.569 & & & 1.000 & & & 0.379 & & & NA \\
\hline Well differentiated & 1 & 10 & & 3 & 5 & & 3 & 9 & & 6 & 22 & & 0 & 0 & \\
\hline Moderately differentiated & 0 & 0 & & 0 & 0 & & 0 & 0 & & 0 & 0 & & 0 & 0 & \\
\hline Poorly differentiated & 2 & 6 & & 1 & 6 & & 4 & 8 & & 10 & 19 & & 0 & 0 & \\
\hline Undifferentiated & 0 & 0 & & 0 & 0 & & 0 & 0 & & 0 & 0 & & 0 & 0 & \\
\hline
\end{tabular}

${ }^{\mathrm{a}} \mathrm{Chi}$-square test or Fisher's exact test.

+ metastasis of SCC to cervical node, - no metastasis of SCC to cervical node. 
and irregular contour of lymph nodes [5]. Morphological MRI criteria have improved the detection of lymph node metastases in head and neck squamous cell carcinoma [4]. However, Feinmesser et al. argued that little advantage was achieved from MRI over clinical examination when detecting metastatic neck disease, and the present size criterion for the diagnosis of occult malignant nodes is not reliable [3].

Many methods have identified prognostic factors of HNSCC. Moreover, the biomarker study for prognostic factors such as MMP-13 [9], COX-2 expression [10], and gene expression (molecular analysis) marker [11] can predict occult metastasis, but the accuracy to detect occult metastasis is inconclusive.

Jan et al. reported the importance of tumor stage, surgical margin status, and extracapsular spread of cervical nodal metastasis as the most important prognostic factors in patients with buccal SCC [20]. Apisarnthanarax et al. found no significant difference between the extent of extracapsular extension and lymph node size. The incidence of extracapsular extension is associated with larger nodal size [21]. However, extracapsular extension is found in a substantial number of nodes with a diameter of $<10 \mathrm{~mm}$ [22]. In our study, we found extracapsular extension $69.55 \%$ and no statistical significance among extracapsular extension of cervical nodes and staging, size of primary tumor, pathologic differentiated, and size of cervical node.

In our study, the size of cervical node ranges from 1 to $40 \mathrm{~mm}$ and the size of cervical node metastasis can be found as small as $2 \mathrm{~mm}$. A statistical significance was found between the size of cervical nodes and the site of primary tumor of the oral tongue and lip $(p<0.05)$. We recommended performing neck dissection in all cases of SCC of the base of the tongue, floor of the mouth, buccal mucosa, and retromolar trigone. According to each site of tumor, the size of cervical lymph node of SCC of the oral tongue was statistically significant with the size of tumor and tumor grading. The size of cervical lymph node of SCC of the lip was statistically significant with clinical staging and tumor grading. However, the size of primary tumor and tumor grading association with the cervical nodes status were inconclusive.

In therapeutic neck dissection, statistically significant associations were found between the size of cervical lymph node and SCC of the oral tongue, lip, and floor of the mouth. In prophylactic neck dissection, statistically significant associations were observed between the size of cervical lymph node and SCC of the oral tongue, base of the tongue, and floor of the mouth. It meant that the larger the primary tumor, the higher the chance of metastasis, while SCC of the base of the tongue, floor of the mouth, buccal mucosa and retromolar trigone had the cervical node metastasis in small primary tumors.
Herein, we recommended performing neck dissection, in cases of SCC of the base of the tongue, floor of the mouth, buccal mucosa, and retromolar trigone.

No statistically significant associations were found among extracapsular extension of the cervical node and clinical staging, size of primary tumor, pathologic differentiation, and size of cervical nodes. No statistically significant associations were observed among lymphovascular invasion of primary tumor, clinical staging, and pathologic differentiation. A statistically significant association was found between the size of SCC at the oral tongue and lymphovascular invasion. The importance of extracapsular extension and lymphovascular invasion is still unclear and further data is needed to support the prognosis.

One disadvantage of this study was that the pathologic status of the cervical node could not be determined in the patients which neck dissection was not performed. Additionally, we did not define the level of cervical nodes, despite the fact that cervical node levels I and II may increase the chance of node metastasis more than cervical node levels III and IV because this was a retrospective review with insufficient data. Moreover, a numerical difference between the tumor sites was observed, due to the incidence of sites of primary tumors was different. We did not study the survival rate of patients but emphasized the size of the metastatic cervical lymph node.

\section{Conclusions}

The status of cervical lymph nodes is one of the prognostic factors in HNSCC. No gold standard exists, except the histopathologic examination to identify nodal status. The size of cervical node metastasis can be found as small as 2 $\mathrm{mm}$. A statistical significance was found between the size of cervical nodes and the site of primary tumor of the oral tongue and lip. Herein, we recommended performing neck dissection in all cases of SCC of the base of the tongue, floor of the mouth, buccal mucosa, and retromolar trigone.

\section{Abbreviations \\ HNSCC: Head and neck squamous cell carcinoma; CT: Computed tomography; MRI: Magnetic resonance imaging; PET: Positron emission tomography; FNA: Fine needle aspiration.}

\section{Competing interests}

The authors declare that they have no competing interests.

\section{Authors' contributions}

$\mathrm{CB}$ designed the study, analyzed and interpreted the data, and contributed to the manuscript writing. WJ collected the data and medical records. MC reviewed the histopathologic data of primary tumors and cervical nodes. All authors read and approved the final manuscript.

\section{Acknowledgements}

The authors thank Mrs. Supak Ukritchon, statistician, and the research assistant of the Office of Research Development in Phramongkutklao College of Medicine for their kind help in the statistical analysis of this article. No sponsors or institutional grants were obtained for this study. This study has not been published previously in any journal. 


\section{Author details}

'Division of Plastic and Reconstructive Surgery, Department of Surgery, Phramongkutklao Hospital and College of Medicine, 315 Ratchawithi Road, Thung Phayathai, Ratchathewi, Bangkok 10400, Thailand. 'Department of Pathology, Phramongkutklao College of Medicine, Bangkok, Thailand.

Received: 29 August 2014 Accepted: 10 January 2015

Published online: 15 February 2015

\section{References}

1. Hung $\mathrm{SH}$, Lin $\mathrm{CY}$, Lee JY, Tseng H. Computed tomography image characteristics of metastatic lymph nodes in patients with squamous cell carcinoma of the head and neck. AurisNasus Larynx. 2012:39:606-10.

2. Wiener E, Pautke C, Link TM, Neff A, Kolk A. Comparison of 16-slice MSCT and MRI in the assessment of squamous cell carcinoma of the oral cavity. Eur J Radiol. 2006;58:113-8.

3. Feinmesser R, Freeman JL, Noyek AM, Birt D, Gullane P, Mullen JB. MRI and neck metastases: a clinical, radiological, pathological correlative study. J Otolaryngol. 1990;19:136-40.

4. de Bondt RB, Nelemans PJ, Bakers F, Casselman JW, Peutz-Kootstra C, Kremer B, et al. Morphological MRI criteria improve the detection of lymph node metastases in head and neck squamous cell carcinoma: multivariate logistic regression analysis of MRI features of cervical lymph nodes. Eur Radiol. 2009;19:626-33.

5. Ding ZX, Liang BL, Shen J, Xie BK, Huang SQ, Zhang B. Magnetic resonance imaging diagnosis of cervical lymph node metastasis from lingual squamous cell carcinoma. Ai Zheng. 2005;24:199-203.

6. Matsubara R, Kawano S, Chikui T, Kiyosue T, Goto Y, Hirano M, et al. Clinical significance of combined assessment of the maximum standardized uptake value of F-18 FDG PET with nodal size in the diagnosis of cervical lymph node metastasis of oral squamous cell carcinoma. Acad Radiol. 2012;19:708-17.

7. Yamazaki Y, Saitoh M, Notani K, Tei K, Totsuka Y, Takinami S, et al. Assessment of cervical lymph node metastases using FDG-PET in patients with head and neck cancer. Ann Nucl Med. 2008:3:177-84.

8. Kagawa T, Yuasa K, Fukunari F, Shiraishi T, Miwa K. Quantitative evaluation of vascularity within cervical lymph nodes using Doppler ultrasound in patients with oral cancer: relation to lymph node size. Dentomaxillofac Radiol. 2011:40:415-21.

9. Mäkinen LK, Häyry V, Atula T, Haglund C, Keski-Säntti H, Leivo I, et al. Prognostic significance of matrix metalloproteinase-2, $-8,-9$, and -13 in oral tongue cancer. J Oral Pathol Med. 2012;41:394-9. doi:10.1111/j.1600-0714.2011.01110.x.

10. Mohammad S, Ram H, Gupta PN, Husain N, Bhatt ML. Overexpression of COX-2 in oral squamous cell carcinoma patients undergoing chemoradiotherapy. Natl J Maxillofac Surg. 2011;2:17-21.

11. Méndez E, Lohavanichbutr P, Fan W, Houck JR, Rue TC, Doody DR, et al. Can a metastatic gene expression profile outperform tumor size as a predictor of occult lymph node metastasis in oral cancer patients? Clin Cancer Res. 2011;17:2466-73.

12. Haksever $M$, Inançlı HM, Tunçel U, Kürkçüoğlu SS, Uyar M, Genç $O$, et al. The effects of tumor size, degree of differentiation, and depth of invasion on the risk of neck node metastasis in squamous cell carcinoma of the oral cavity. Ear Nose Throat J. 2012;91:130-5.

13. Shah JP, Candela FC, Poddar AK. The patterns of cervical lymph node metastases from squamous carcinoma of the oral cavity. Cancer. 1990:66:109-13.

14. Kuperman DI, Auethavekiat V, Adkins DR, Nussenbaum B, Collins S, Boonchalermvichian C, et al. Squamous cell cancer of the head and neck with distant metastasis at presentation. Head Neck. 2011:33:714-8. doi:10.1002/hed.21529.

15. Alkureishi LW, Ross GL, MacDonald DG, Shoaib T, Gray H, Robertson G, et al. Sentinel node in head and neck cancer: use of size criterion to upstage the no neck in head and neck squamous cell carcinoma. Head Neck. 2007;29:95-103.

16. Ozer F, Ozer C, Erkan AN, Yavuz H. The therapeutic role and effectiveness of selective neck dissection in the management of NO neck. Kulak Burun Bogaz Ihtis Derg. 2009;19:192-7.

17. Di B, Li XM, Shao YL, He ZG. Efficacy of radical neck dissection for advanced cervical nodal metastases. Zhonghua Er Bi Yan Hou Tou Jing Wai Ke Za Zhi. 2005:40:106-10.
18. Broglie MA, Haerle SK, Huber GF, Haile SR, Stoeckli SJ. Occult metastases detected by sentinel node biopsy in patients with early oral and oropharyngeal squamous cell carcinomas: impact on survival. Head Neck. 2013;35:660-6. doi: 10.1002/hed.23017

19. Kowalski LP, Sanabria A. Elective neck dissection in oral carcinoma: a critical review of the evidence. Acta Otorhinolaryngol Ital. 2007:27:113-7.

20. Jan JC, Hsu WH, Liu SA, Wong YK, Poon CK, Jiang RS, et al. Prognostic factors in patients with buccal squamous cell carcinoma: 10-year experience. J Oral Maxillofac Surg. 2011;69:396-404.

21. Apisarnthanarax S, Elliott DD, El-Naggar AK, Asper JA, Blanco A, Ang KK, et al. Determining optimal clinical target volume margins in head-and-neck cancer based on microscopic extracapsular extension of metastatic neck nodes. Int J Radiat Oncol Biol Phys. 2006;64:678-83.

22. Ghadjar P, Schreiber-Facklam H, Gräter R, Evers C, Simcock M, Geretschläger A, et al. Quantitative analysis of extracapsular extension of metastatic lymph nodes and its significance in radiotherapy planning in head and neck squamous cell carcinoma. Int J Radiat Oncol Biol Phys. 2010;76:1127-32. doi:10.1016/j.jijrobp.2009.03.065.

\section{Submit your next manuscript to BioMed Central and take full advantage of:}

- Convenient online submission

- Thorough peer review

- No space constraints or color figure charges

- Immediate publication on acceptance

- Inclusion in PubMed, CAS, Scopus and Google Scholar

- Research which is freely available for redistribution

Submit your manuscript at www.biomedcentral.com/submit
C Biomed Central 